\title{
Comparison of Domain Specific Connectivity Metrics for Estimation Brain Network Indices in Boys With ADHD-C
}

SERAP AYDIN ( $\nabla$ drserapaydin@hotmail.com )

Hacettepe Universitesi Tip Fakultesi https://orcid.org/0000-0002-4026-0750

Fatih Hilmi CETIN

Selçuk Üniversitesi Tip Fakültesi: Selcuk Universitesi Tip Fakultesi

Merve UYTUN

Ankara University Faculty of Medicine: Ankara Universitesi Tip Fakultesi

Zehra BABADAG

Kayseri Training and Research Hospital: Kayseri Sehir Egitim ve Arastirma Hastanesi

Ahmet Sami GUVEN

Necmettin Erbakan Üniversitesi Meram Tıp Fakültesi: Necmettin Erbakan Universitesi Meram Tip

Fakultesi

Yasemen ISIK

Gazi University Faculty of Medicine: Gazi Universitesi Tip Fakultesi

\section{Research Article}

Keywords: Pearson Correlation, Brain Connectivity, Graph Theory, EEG, ADHD

Posted Date: August 31st, 2021

DOl: https://doi.org/10.21203/rs.3.rs-822995/v1

License: (c) (i) This work is licensed under a Creative Commons Attribution 4.0 International License.

Read Full License

Version of Record: A version of this preprint was published at Biomedical Signal Processing and Control on July 1st, 2022. See the published version at https://doi.org/10.1016/j.bspc.2022.103626. 


\title{
Comparison of Domain Specific Connectivity Metrics for Estimation Brain Network Indices in Boys with ADHD-C
}

\author{
Serap Aydın - Fatih Hilmi Çetin . \\ Merve Çıkılı Uytun, · Zehra Babada g̃ • \\ Ahmet Sami Güven · Yasemen Işık
}

Received: date / Accepted: date

\begin{abstract}
The study includes Graph Theoretic advanced EEG analysis in order to investigate the impact of pharmacological treatment with osmotic release oral system-methylphenidate for a month in 18 boys (aged between 7-12 years) with Attention-Deficit-Hyperactivity Disorder, combined type. In analysis, neurofunctional dependency levels across the cortex are estimated by using six different connectivity estimation approaches based on three separate domains such as time (Pearson Correlation, Spearman Correlation), frequency

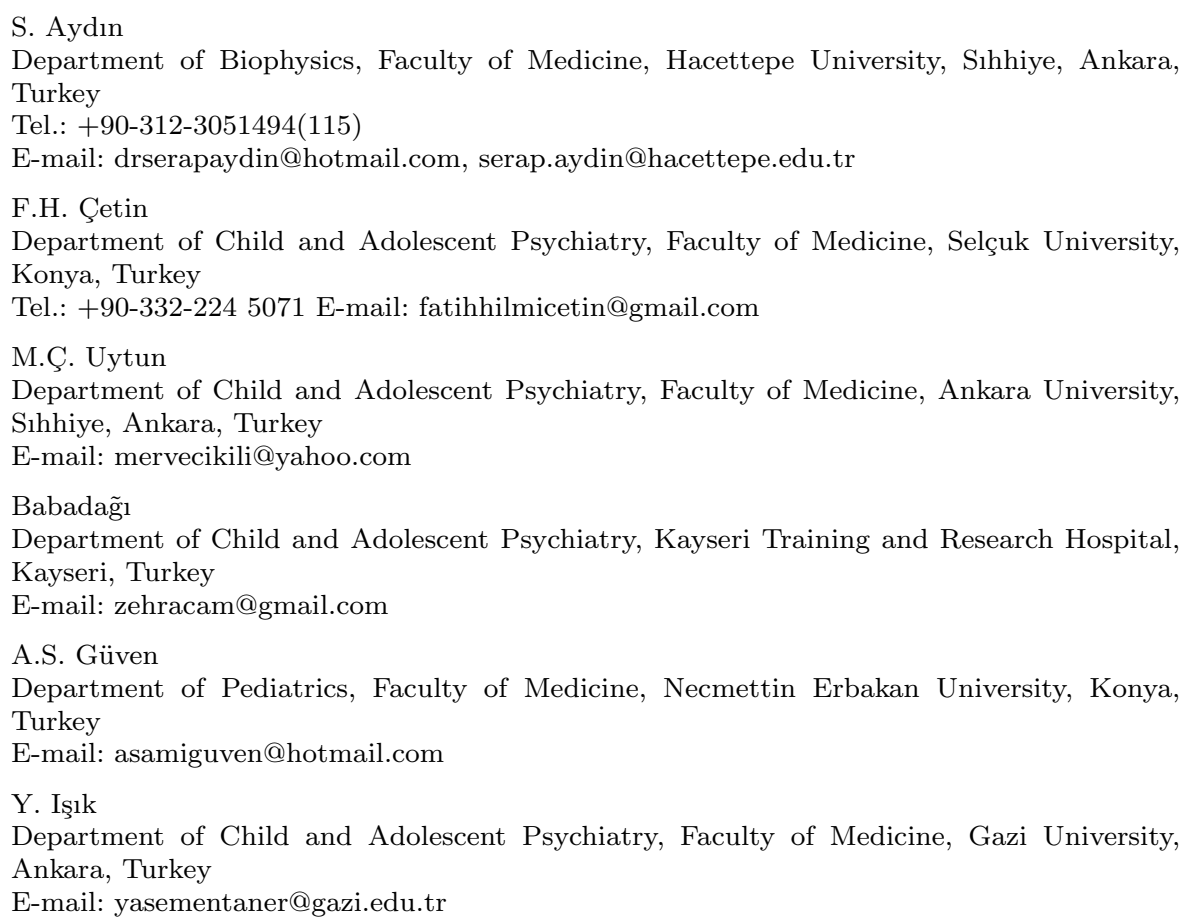


(Directed Transfer Function, Partial Directed Coherence) and phase (Phase Locking Value, Phase Lag Index). All methods are examined for eyes-opened resting-state EEG segments of $2 \mathrm{sec}$ over a single trial of $1 \mathrm{~min}$ in both preand post-treatment recordings. Then, five quantitative brain network indices of transitivity, clustering coefficients, assortativity, global efficiency and modularity are computed from connectivity estimations.

Performance of connectivity estimators are compared to each other with respect to two-class classifications (pre-treatment features vs post-treatment features) by using Support Vector Machines driven by brain network indices. The highest classification accuracy of $80.74 \%$ is obtained with Pearson Correlation. Statistical one-way Anova test, pair-wise multiple comparison test and step-wise logistic regression modelling are all used to observe the most sensitive network index estimated from Pearson Correlations. In addition to quantitative results, statistical box-plots of the estimated network indices are shown graphically. When modularity index is excluded from the features, classification accuracy is increased to $83.79 \%$. Overall results reveal that brain segregation and resilience are increased by the treatment. In particular, the most meaningful brain network measures can be estimated from time domain statistical correlations between resting-state eyes open EEG segments in order to understand the alterations in neuronal transmission mechanism across the cortex in response to a specific treatment in pediatric psychiatry.

Keywords Pearson Correlation · Brain Connectivity · Graph Theory · EEG · ADHD

\section{Introduction}

Attention Deficit Hyperactivity Disorder (ADHD) can be defined as a dysfunction characterized by difficulty in attentional maintenance and/or by excessive hyperactivity and impulsivity [1]. Depending on the dominant symptoms, three subtypes of ADHD are considered in diagnose as follow: inattentive is predominant in ADHD-I, hyperactive-impulsive is predominant in ADHD-HI, and existence of both attentional maintenance and excessive hyperactivity in combination with impulsivity in ADHD-C [1]. Both functional magnetic resonance imaging (fMRI) slices [2-4] and resting-state EEG recordings $[5,6]$ have been analyzed to show impaired functional connectivity (FC) between different brain regions in diagnose of ADHD as compared to healthy controls. Mostly, spectral coherence was used to quantify functional connectivity, however, the findings of the studies differ from each other depending on whether EEG recording pairs are near or far from to each other (intra or inter-hemispheric) [7-9]. As well, indicator is pairwise estimation in several studies, while the grand average is considered in the others. Moreover, the other parameters that cause the differences in results are non-identical clinical scores and gender-age distributions of the participants.

In Graph Theory, the brain is assumed to be a complex network. Then, both structural and functional brain network measures can be computed through 
neuroimaging and electrophysiological research studies. The researchers have begun to use EEG based brain connectivity estimations in terms of network parameters explained by Graph Theory in various neuro-psychiatric and cognitive disorders [12-16]. The recording electrode placements and neuronal pathways between electrode pairs are constituted by nodes and edges, respectively in theoretical manner. Mostly, functional interactions across recording sites have been estimated using Pearson Correlation (PC) approach due to its sensitivity to indirect, "third-party" effects that arise from correlation between two nodes (EEG recording sites) in the absence of a direct anatomical connection since they are both connected to a third, intermediary node. Examining PC provides both in-phase coupling (high correlation) and out-of-phase relationship (negative correlation) between pairs of simultaneously measured time series. In case of no linear relationship, PC becomes zero. PC can also measure the effect of a common driving stimulus, or post-synaptic potential variations $[17,18]$. Thus, use of PC in estimating correlation matrix of the cortex, brain network measures can be artificially inflated [19]. Moreover, PC does not distinguish between phase coupling and amplitude coupling modes hypothesized to underlie brain function.

The other metrics used to determine connectivity matrix are spectral Coherence (COH), Phase Locking Value (PLV) and Phase Lag Index (PLI) [20]. $\mathrm{COH}$ is a linear and non-causal synchronization measure based on neural information transfer between two time series. $\mathrm{COH}$ is sensitive to volume conduction in EEG analysis due to coherent oscillations between neuronal populations [21]. The estimated $\mathrm{COH}$ values can quantify the extent to which EEG recordings share a constant oscillating frequency and phase difference ranging anywhere from zero (in-phase) to 180 degrees (anti-phase). In this case, two EEG recordings have a squared magnitude coherence ranging from 1 (in-phase: sharing the same oscillation frequency) to 0 (anti-phase). PLV can be considered as derivation on $\mathrm{COH}$ estimations. Thus, PLV clearly differs from $\mathrm{COH}$ in the sense that PLV reduces volume conduction effect through dividing the phase and amplitude components in power spectra of EEG series. In other words, estimation of PLV is a non-linear functional connectivity method that does not require stationary assumption of EEG series. Therefore, PLV provides the phase locking of each latency between two EEG recordings [22]. Since a consistent, nonzero phase lag between two EEG recordings is not sensitive to the influence of volume conduction from a single strong source, PLI has been proposed as superior statistical dependency metric [23]. PLI can provide true interactions between two EEG recording sites (cortical regions), and, PLI estimations can clearly differentiate EEG electrode pairs with either strong coupling $\left(\mathrm{PLI}_{¿} 0\right)$ or without coupling (PLI 0). Therefore, PLI has been mostly used in clinical evaluations such as investigation of repeated transcranial direct current stimulation (tDCS) effect on refractory epilepsy [28], estimation of Autism Spectrum Disorder (ASD) neurophysiological phenotype in infants [25] and estimation of biomarkers for brain maturation [26]. In recent neuroscience works, PLI has been used for estimation of brain network measures from hemispheric dependency matrixes in comparing the controls 
with the patients in several disorders such as schizophrenia [27], dyslexia [40], and major depression [41]. In these studies, direction of information flow and causality are not considered. Indeed, they can provide integration and segregation of brain networks in well temporal resolution.

Different from those hemispheric dependency methods, Partial Directed coherence (PDC) and Directed Transfer Function (DTF) have been proposed as effective connectivity metrics in estimating brain network indices based on Graph Theory. PDC is examined to understand brain network mechanism in different mental tasks in healthy populations [36], while, DTF is applied to both EEG recording and fMRI recordings in order to observe the effects of trans-cranial stimulations in neuropsychiatric disorders such as major depression and bipolar disorder [37]. The common concepts between PDC and DTF are that both methods are based on Granger causality where EEG epochs are assumed to be modeled by multivariate autoregressive (MVAR) model in characterizing effective connections over cortex. Both methods (PDc and DTF) are applied to surface EEG recordings for detection of paranoid schizophrenia in recent reference study [38].

The recent computational neuroscience studies including patients with ADHD, show functional brain connectivity alterations in ADHD by means of decreased global efficiency and increased shortest path length based on Graph Theory $[35,39]$. In previous several neuroimaging studies, the findings reveal that brain connectivity measures are changed at mostly temporal and frontal regions in patients with both inattention and hyperactivity $[29,30]$. In the present study, both statistical (PC and $\mathrm{SC}$ ) and phase domain dependency methods in addition to effective connectivity approaches (DTF and PDC) have been examined to obtain neurofunctional improvements caused by OROS-MPH in terms of quantitative brain network measures (transitivity, assortativity, clustering coefficients, global efficiency, modularity) in boys with ADHD-C. Treatment with OROS-MPH is the most commonly prescribed medication for children with ADHD in many countries. This is the first study that compares the performances of statistical correlations with phase domain functional dependency and frequency domain effective connectivity estimations in terms of graph theoretic brain network indices in extracting the impact of OROS-MPH for a month in boys. The results are evaluated by examining not only classifiers but also statistical tests. As well, step-wise logistic regression modelling is also applied to estimated brain network indices. Finally, statistical box-plot distributions are shown to present the functional alterations induced by OROS-MPH.

\section{Methods}

The patients were diagnosed by the second author at Department of Child and Adolescent Psychiatry at Emel-Mehmet Tarman Children Hospital in Kayseri, Turkey in between 2016 and 2017. Among the patients, the identical treatment was osmotic release oral system-methylphenidate (OROS-MPH) for a 


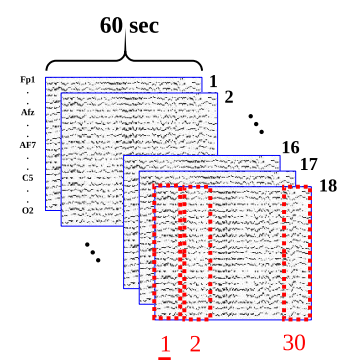

\# of subject $=18 \quad(k=1, \ldots, 18)$

For each subject:

\# of EEG recording channels $=19(\mathrm{chn}=1, \ldots, 19)$

the length of single trial $=1 \mathrm{~min}$

the length of short EEG epoch $=2 \mathrm{sec}$

\# of epochs in analysis interval $=\mathbf{3 0}(\mathrm{i}=1, \ldots, 30)$

For each epoch pair:

Dimension of EEG dependency matrix $\left(D_{k, j}\right)=19 \times 19$

Threshold in transforming $\left(\mathrm{D}_{\mathrm{k}, \mathrm{i}}\right)$ to adjacency matrix $\left(\mathrm{A}_{\mathrm{k}, \mathrm{i}}\right)=60 \% \max \left(\mathrm{D}_{\mathrm{k}, \mathrm{i}}\right)$

Fp1
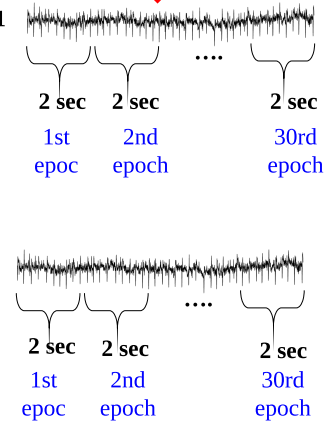

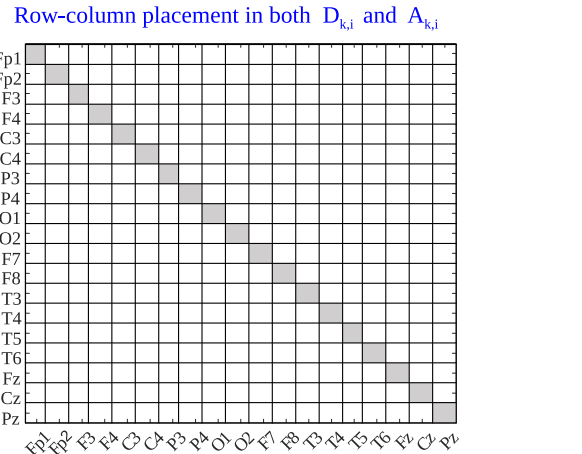

Fig. 1 Main steps in EEG analysis in pre- and post-treatment recordings: Six methods are comparatively examined to obtain brain dependency estimations

month. The volunteers took medication at every morning after breakfast. Pretreatment and post-treatment brain activities of them were collected from scalp surface at eyes-opened resting-state in light and sound controlled room. Ethical approval for this study was obtained from the Ethics Committee of Erciyes University, Medical Faculty by second author of the study. The Ethical Approval Number is 2016/422 on July, 15 in 2016. EEG data was collected from patients to investigate possible effects of OROS-MPH on brain activities in terms of EEG analysis. EEG analysis principle is shown in Figure 1.

\subsection{The Participants and Clinical Evaluation}

Right handed 18 boys with mean age of 10.27 (between 7 and 12) were diagnosed with ADHD-C in this study. As primary clinical evaluation, expert physicians used the diagnostic criteria of the Diagnostic and Statistical Manual of Mental Disorders 4rd Edition (DSM-IV-TR) to interview with the patients. As secondary interview, Turkish Version of Schedule for Affective Disorders and Schizophrenia for School-Age Children-Present and Lifetime Version (KSADS-PL-T), a semi-structured interview tool described in reference [33], was followed by the physicians. Both validity and reliability of this tool was examined in Turkish as presented in reference [34]. As supportive clinical tool, 
Turgay DSM-IV Disruptive Behavior Disorders Rating Scale (T-DSM-IV-S) was attempted. The T-DSM-IV-S, described in reference [31] was translated and adapted into Turkish as presented in reference [32]. Table 1 shows the patients' clinical scores in accordance with T-DSM-IV-S in addition to their age. The listed scores show severity of the symptoms on a 4-point Likert-type scale $(0=$ not at all, $1=$ just a little, $2=$ much, $3=$ very much). Psychiatrist having the relevant certification (the first author of the study) interviewed the parents of the boys with respect to this tool.

Table 1 Clinical evaluation of the patients in pre- and post-treatment periods with respect to T-DSM-IV-S (IA: inattention, HI: hyperactivity-impulsivity, ODD: Opposition defiant disorder, CD: Conduct Disorder, S: Total score)

\begin{tabular}{ll|rlrrr||rrrrr} 
& & \multicolumn{9}{|c}{ pre-treatment } \\
\hline patient-no & age & IA & HI & ODD & CD & S & IA & HI & ODD & CD & S \\
\hline \hline 1 & 9 & 13 & 20 & 9 & 0 & 42 & 6 & 9 & 6 & 0 & 21 \\
2 & 12 & 20 & 22 & 14 & 1 & 57 & 11 & 10 & 8 & 1 & 30 \\
3 & 9 & 14 & 18 & 6 & 1 & 39 & 6 & 8 & 4 & 1 & 19 \\
4 & 8 & 19 & 22 & 8 & 4 & 53 & 9 & 9 & 5 & 3 & 26 \\
5 & 10 & 20 & 22 & 8 & 0 & 50 & 10 & 10 & 6 & 0 & 26 \\
6 & 9 & 16 & 10 & 5 & 0 & 31 & 6 & 6 & 3 & 0 & 15 \\
7 & 7 & 14 & 13 & 7 & 0 & 34 & 9 & 9 & 6 & 0 & 24 \\
8 & 10 & 20 & 17 & 4 & 1 & 42 & 9 & 8 & 4 & 1 & 22 \\
9 & 7 & 19 & 18 & 8 & 1 & 46 & 6 & 9 & 6 & 1 & 22 \\
10 & 7 & 14 & 13 & 12 & 3 & 42 & 6 & 7 & 10 & 2 & 25 \\
11 & 9 & 23 & 17 & 12 & 2 & 54 & 12 & 8 & 8 & 2 & 30 \\
12 & 10 & 22 & 17 & 14 & 3 & 56 & 10 & 8 & 6 & 2 & 26 \\
13 & 7 & 15 & 10 & 8 & 2 & 35 & 10 & 6 & 6 & 2 & 24 \\
14 & 10 & 24 & 16 & 8 & 3 & 51 & 11 & 4 & 6 & 2 & 23 \\
15 & 10 & 23 & 15 & 8 & 0 & 46 & 11 & 3 & 7 & 0 & 21 \\
16 & 7 & 10 & 10 & 7 & 0 & 27 & 3 & 6 & 5 & 0 & 14 \\
17 & 8 & 26 & 27 & 15 & 3 & 71 & 12 & 10 & 10 & 2 & 34 \\
18 & 8 & 11 & 16 & 6 & 0 & 33 & 4 & 4 & 4 & 0 & 12 \\
\hline
\end{tabular}

Table 2 shows the changes in clinical scores induced by treatment with OROS-MPH in patients.

Table 2 The changes on five clinical scores with T-DSM-IV-S in the patients (IA: inattention, HI: hyperactivity-impulsivity, ODD: Opposition defiant disorder, CD: Conduct Disorder, tS: total Score)

\begin{tabular}{l|ccc} 
& Pre-treatment & Post-treatment & Change in Score \\
\hline \hline T-DSM-IV-S IA & $17.94 \mp 4.72$ & $8.38 \mp 2.78$ & $9.55 \mp 2.72$ \\
T-DSM-IV-S HI & $16.83 \mp 4.68$ & $7.44 \mp 2.17$ & $9.38 \mp 3.72$ \\
T-DSM-IV-S ODD & $8.83 \mp 3.22$ & $6.11 \mp 1.93$ & $2.72 \mp 1.93$ \\
T-DSM-IV-S CD & $1.33 \mp 1.37$ & $1.05 \mp 0.99$ & $0.27 \mp 0.46$ \\
T-DSM-IV-S TS & $44.94 \mp 11.10$ & $23.00 \mp 5.65$ & $21.94 \mp 6.91$
\end{tabular}




\subsection{EEG Data Collection}

19-channel eyes-opened surface EEG series were recorded from awake boys seating on a comfortable chair in air-conditioned and light controlled room at early in the morning. EEG data acquisition system was Nihon Kohden Model (1200K, Tokyo, Japan). Ag-AgCl plated disk electrodes were placed on scalp with respect to international 10-20 electrode placement system. The reference electrodes were A1 and A2. Electrodes' impedances were kept to below $5 k \Omega$. Sampling frequency was $200 \mathrm{~Hz}$. The ground electrode was midway between Fpz and Fz. The recordings with muscular and ocular artifacts were preliminarily identified by an automatic computerized procedure.

Schematic representation of EEG analysis procedure is shown in Figure 1. Considering each electrode pair, short EEG segments of $2 \mathrm{sec}$ were filtered by using an Infinite-Impulse-Response (IIR) filter with order of 35 in order to remove network noise at $60 \mathrm{~Hz}$ (Notch filtering). Later, Finite Impulse Response (FIR) filter was applied as secondary filtering operation with the following parameters as: filter order $=35$, density factor $=20$, wo $=60 / \mathrm{Fs} / 2$, bandwidth $(\mathrm{BW})=\mathrm{wo} / 354$, pass-band ripple $=0.05750$, stop-band attenuation $=0.0001,1 \mathrm{st}$ stop-band attenuation $=0.001,1$ st stop-band and pass-band frequencies are $0.5 \mathrm{~Hz}$ and $1 \mathrm{~Hz}$, 2nd pass-band and stop-band frequencies are $40 \mathrm{~Hz}$ and 40.5 $\mathrm{Hz}$. The filtered EEG data $(200 \mathrm{~Hz})$ were primarily segmented off-line into consecutive non-overlapped epochs of $2 \mathrm{~s}$.

\subsection{Neuro-cortical Dependency Methods}

Considering two EEG segments ( $\mathrm{X}$ and $\mathrm{Y}$ ) as random variables with mean values $\left(\mu_{x}, \mu_{y}\right)$ and standard deviations $\left(\sigma_{x}, \sigma_{y}\right)$, Pearson Correlation (PC) provides a linear statistical measurement to quantify linear dependency between two EEG recording sites in form,

$$
P C_{x y}=\frac{1}{N} \sum_{k=1}^{N} \frac{\left(x_{k}-\mu_{x}\right)\left(y_{k}-\mu_{y}\right)}{\sigma_{x} \sigma_{y}}
$$

$\mathrm{PC}$ gives a normalised measurement of the covariance ranged -1 and +1 (perfect relationship) where zero means no association. Application of PC to rank-transformed data is defined as Spearman Correlation (SC) given by,

$$
S C_{x y}=\frac{\left(x_{k}-\mu_{x}\right)\left(y_{k}-\mu_{y}\right)}{\sqrt{\sum_{k=1}^{N}\left(x_{k}-\mu_{x}\right)^{2}} \sqrt{\sum_{k=1}^{N}\left(y_{k}-\mu_{y}\right)^{2}}}
$$

Similar to PC, SC provides the values from +1 (perfect association of ranks) to -1 (perfect negative association of ranks). In case of the weaker association between the ranks, SC closer to zero. The nonlinear dependency metrics, PLV and PLI are used to estimate phase relations between two random variables $(x$ and $y)$ PLV is defined by following equation, 


$$
P L V_{x y}^{i}=\frac{1}{N} \exp j\left(\phi_{x}-\phi_{y}\right)
$$

where $\phi_{x}$ and $\phi_{y}$ denote phase courses of $x$ and $y$, respectively. Regarding consistent phase difference $\left(\Delta \phi=\phi_{x}-\phi_{y}\right)$, PLV takes +1 (well-synchrony). If there is no phase-relationship between two series, PLV gives zero due to random phase difference. Therefore, PLV can be considered as a measure of segment-to-segment variability in terms of relative phase difference between two EEG segments.

PLI is introduced to obtain reliable estimates of phase synchronization that are invariant against the presence of common sources, i.e. volume conduction. Thus, PLI is given by following equation for each pair of EEG segments,

$$
P L I_{x y}=|\langle\operatorname{sign}[\triangle \phi]\rangle|
$$

where $\triangle \phi$ denotes the phase difference between X and Y. The PLI can provides the values from 0 (either no coupling or coupling with a phase difference centered around 0 ) to 1 (perfect phase locking at a value of $\triangle \phi$ ). Non-zero values refer the stronger phase relations.

DTF can be described as an estimator of effective connectivity, based on Multivariate Autoregressive Mode (MVAR) [46]. A causal influence of two EEG channels $(x$ and $y)$ is computed in form,

$$
D T F_{x y}=\frac{|H(f)|^{2}}{\sum_{m=1}^{k}\left|H_{x, m}(f)\right|^{2}}
$$

Here, $H(f)$ refers the transfer function of the MVAR model in accordance with the frequency of $f$. Depending on its computational procedure, DTF produces spectral characteristics, i.e. frequency domain properties of all EEG channels, but not only input channel $(x)$.

PDC is presented to measure directed coherence between pairs of a structures. Rather then merely describing mutual synchrony between EEG series, PDC can provide whether and how two locations are functionally connected in frequency domain in form,

$$
P D C_{x y}=\frac{A_{x y}(f)}{\sqrt{a_{y}^{*}(f) a_{y}(f)}}
$$

where $A_{x y}(f)$ indicates Fourier transform of MVAR model coefficients. $a_{y}(f)$ refers column of matrix determined from estimations in $A_{x y}(f)$. The notation of $*$ denotes the transpose and complex conjugate operation.

Both DTF and PDC are performed by using open source software package called eConnectome2.0 toolbox introduced in reference [47]. The other methods are implemented by using both signal processing and statistical \& machine learning toolboxes in Matlab2020Rb. 


\subsection{Brain Network Measures}

Brain Connectivity Toolbox (BCT) that was introduced in the reference [42] was used to estimate brain network measures based on the following concepts:

Segregation quantifies the presence of densely interconnected cortical regions mentioned as clusters or modules of the network. Clustering Coefficients (CC) is assigned as a segregation measure in Graph Theory. CC describes the tendency of the nodes to form locally triangles (connected edges) across the node's neighbors that are also neighbors of each other at different layers. The other segregation measure is Transitivity $(\mathrm{T})$ that is a variant of CC. Assortativity is network resilience (r) measure meaning the extent of degreedegree correlations present in the network topology.

Efficiency/Integration Global Efficiency (GE) depends on the number of disconnected nodes of a graph. The resulting GE is primarily influenced by short paths, while LE is the average efficiency of information flow between close neighborhoods within a local sub-graph.

Modularity (Q) means the degree to which the network tends to segregate into relatively independent modules as subnetworks. Q reflects the capacity of a network to support functional sub-specialization.

The presence of densely interconnected clusters is associated with functional segregation in an anatomical network. However, the presence of clusters is associated with statistical dependencies of segregated neural processing in a functional network. As a quantitative metric of segregation, CC is obtained as the average clustering coefficients over all nodes in the network. In other words, CC refers the fraction of the edges around a node (the fraction of the node's neighbors that are also neighbors of each other). Therefore, the higher $\mathrm{CC}$ indicates the increased efficiency of information flow in cortical sub-regions depending on the prevalence of clustered connectivity around individual nodes. The connectivity of a region to its neighbors is measured as transitivity.

Preferentially connection of the nodes of similar degree is called as assortative network. In case of connections between dissimilar nodes is named as disassortative. Thus, assortative networks can be assumed to have a stronger tendency to disintegrate into sub-graphs. In assortative networks, EEG recording sites, i.e. nodes tend to be closely interconnected thereby forming a resilient core, which may allow the transmit of information over the whole cortex as a network.

As a functional integration measure, GE will be zero in case of disconnected nodes due to lack of information flow. The lower GE refers the weaker connections between cortical regions. LE estimations range from 0 to 1 (maximum local efficiency in the network due to segregated neural processing). Computationally, quantitative measures of integration is global-efficiency $(G E)$ in form,

$$
G E=\frac{1}{N(N-1)} \sum_{i, j=1, i \neq j}^{N} \frac{1}{d_{i j}}
$$


Here, $d_{i j}$ refers the distance. Quantitative measures of segregation are transitivity $(T)$ and clustering coefficients $C C$ are given in following equations,

$$
T=\frac{\sum_{i=1}^{N} 2 t_{i}}{\sum_{i=1}^{N} k_{i} k_{i-1}} C C=\frac{1}{N} \sum_{i=1}^{N} \frac{2 t_{i}}{k_{i} k_{i-1}} \delta_{m i, m j}
$$

Here, $G_{i}$ refers the subgraph formed by small number of connected nodes where $i$ refers the node and $t_{i}$ refers the number of triangles around the node. $k_{i}$ is used to indicate the network degree meaning of the number of links connected to a node, $i$.

When two nodes ( $i$ and $j$ ) are neighbors, the corresponding connection status will be assigned as $a_{i j}=1$ meaning existence of a link between $i$ and $j$. Conversely, lack of link is presented with $a_{i j}=0$. Therefore, the number of triangles around a node $(i)$ is defined as follows,

$$
t_{i}=\frac{1}{2} \sum_{j, h=1}^{N}\left(a_{i j} a_{i h} a_{j h}\right)
$$

Regarding functional modules of brain network in terms of cortical regions, the strength of their division is quantified by modularity index $(Q)$ in from,

$$
Q=\frac{1}{l} \sum_{, j i=1}^{N}\left(A_{i j}-\frac{k_{i} k_{j}}{l}\right)
$$

Here, $l=\sum_{i, j=1}^{N} A_{i j}$ is the number of edges, $m i$ refers the module where $\delta_{m i, m j}=1$ if $m i=m j$ and $\delta_{m i, m j}=0$ otherwise.

$$
T=\frac{\operatorname{sum}_{i=1}^{N} 2 t_{i}}{\operatorname{sum}_{i=1}^{N}\left(k_{i} k_{i-1}\right)}
$$

Assortativity coefficient $(r)$ is presented as resilience measure in form,

$$
r=\frac{\frac{1}{L} \operatorname{sum}_{i, j=1}^{L} k_{i} k_{j}-\left(\frac{1}{L} \sum_{i, j=1}^{L} \frac{1}{2}\left(k_{i}+k_{j}\right)\right)_{2}}{\operatorname{frac} 1 L \sum_{i, j=1}^{L} \frac{1}{2}\left(k_{i}^{2}+k_{j}^{2}\right)-\left(\frac{1}{L} \sum_{i, j=1}^{L} \frac{1}{2}\left(k_{i}+k_{j}\right)\right)_{2}}
$$

\subsection{Classification of Groups with Support Vector Machines}

Two-class classification schema was examined to discriminate surface EEG measurements recorded from patients just before treatment (Group-1) with leading treatment with OROS-MPH from that of post-treatment (Group-2). Six different hemispheric dependency methods (PC, SC, PLV, PLI, DTF, PDC) were applied to pairs of short segments across 19-channel eyes-opened resting state EEG series of $1 \mathrm{~min}$. Since the length of identical segments was 
2 sec, 30 connectivity matrixes were obtained for each individual. Thus, the number of features was 540 ((\# of subject) $\mathrm{x}$ (\# of segments) $=18 \times 30$ ) in each group for each brain network measure $(T, C C, r, G E, Q)$, computed for each connectivity matrix. When all measures were considered in classifications, the number of features were increased as five times 540. The labels were 0 and 1 for Group-1 and Group-2, respectively. Statistical and Machine Learning toolbox was used in Matlab2020Rb. SVMs were examined with both Radial Basis Functions (RBF) and Gaussians as kernels. The corresponding performances were given with Classification Accuracy (CA) in percent.

\section{Results}

As primary step of the study, the groups were classified through SVM models with respect to the feature set including all brain indices together $(T, C C, r$, $G E$, and $Q$ ) extracted from 30 non-overlapped short EEG segments of $2 \mathrm{sec}$ into single trials of $1 \mathrm{~min}$. The corresponding performance results were summarized in Table 3 where six different dependency approaches were compared to each other with respect to $\mathrm{CA} \%$ in discriminating the groups. Table 3 showed that the highest $\mathrm{CA}$ was provided by $\mathrm{PC}$ with kernels of RBFs with respect to large feature set.

Table 3 Performance results (CA in \%) of SVM classifiers with different kernels driven by brain network measures estimated using PC, SC, PLV, PLI, DTF and PDC

\begin{tabular}{l|c|c|c|c|c|c} 
kernels & PC & SC & PLV & PLI & DTF & PDC \\
\hline \hline RBF & $\mathbf{8 0 . 7 4}$ & 78.42 & 76.85 & 57.68 & 62.77 & 60.54 \\
GF & 79.81 & 77.96 & 76.20 & 57.59 & 59.53 & 58.22 \\
\hline
\end{tabular}

Since, PC provided superior results in comparison to other cortical dependency approaches, step-wise logistic regression modelling was applied to brain network measures in order to highlight the most meaningful measures that sensitive to treatment with OROS-MPH on resting-state neuro-cortical activities of the patients. The resulting coefficients were given in Table 4, where the number of observations is 1080 , error degrees of freedom is 1075, R-squared is 0.207, Adjusted R-Squared is 0.204 and, F-statistic vs. constant model is 70.3. Table 4 showed that modularity did not affected by treatment with OROS$\mathrm{MPH}$, while the other brain network indices were significantly affected.

One-way Anova test and multiple comparison test were used to obtain statistical differences between groups in terms of statistical factors in brain network indices estimated by using PC. The corresponding results are listed in Table 5. In general, one-way Anova is primarily used to test the null hypothesis that all group means are equal, then, multiple comparison test is applied to resulting statistics in order to display an interactive graph of the estimates and comparison intervals [45]. In the present study, the results from these two tests 
Table 4 Step-wise logistic regression modelling results in comparing groups with respect to brain network measures obtained by $\mathrm{PC}$

\begin{tabular}{l|l|ccc} 
measure & estimate & t-stat & SE & p-value \\
\hline \hline intercept & 5.8034 & 0.5718 & 10.14 & $3.5 \mathrm{e}-23$ \\
$\mathrm{~T}$ & -4.49 & 0.4295 & -10.47 & $1.7 \mathrm{e}-24$ \\
$\mathrm{CC}$ & 0.9004 & 0.3321 & 2.71 & 0.0068 \\
$\mathrm{r}$ & -0.1468 & 0.0681 & 2.15 & 0.0314 \\
$\mathrm{GE}$ & -2.9617 & 0.4447 & -0.665 & $4.3 \mathrm{e}-11$ \\
\hline
\end{tabular}

were compatible and supportive to each other. In particular, modularity index, $Q$ was not included in step-wise logistic regression modelling given in Table 4 . The other four indices ( $T, C C, r, G E)$, included in step-wise logistic regression modelling, provided significant and meaningful statistical differences with low p-values.

Table 5 Statistical test results between groups with respect to brain network measures obtained by PC (In one-way Anova tests, SS: Sum of squares due to each source, MS: Mean squares for each source (the ratio between $\mathrm{SS}$ and the degree of freedom), F: F-statistic (the ratio of the mean square), Prob¿F: p-value. In multiple comparisons, 1st: lower limit for $95 \%$ confidence interval, 2nd: difference between group means, 3rd: upper limit for $95 \%$ confidence interval, 4 th: p-value for a hypothesis test that the corresponding mean difference is equal to zero)

\begin{tabular}{l||llll|llll}
\multicolumn{1}{l||}{} & \multicolumn{4}{c}{ one-way Anova test } & \multicolumn{3}{c}{ pairwise multiple comparison test } \\
& SS & MS & F & Prob¿F & 1st & 2nd & 3rd & 4rd \\
\hline \hline T & 0.9066 & 0.9066 & 211.03 & $8.4 \mathrm{e}-44$ & -0.0657 & -0.0579 & -0.0501 & $0.1 \mathrm{e}-9$ \\
$\mathrm{CC}$ & 0.0911 & 0.0911 & 37.44 & $1.3 \mathrm{e}-09$ & -0.0242 & -0.0183 & -0.0124 & $0.1 \mathrm{e}-9$ \\
$\mathrm{r}$ & 8.6883 & 8.6882 & 138.62 & $3.4 \mathrm{e}-30$ & -0.2092 & -0.1793 & -0.1495 & $0.1 \mathrm{e}-10$ \\
$\mathrm{GE}$ & 0.1224 & 0.1224 & 42.63 & $1.01 \mathrm{e}-10$ & 0.0149 & 0.0212 & 0.0276 & $0.1 \mathrm{e}-10$ \\
$\mathrm{Q}$ & 0.0965 & 0.0965 & 18.56 & $1.7 \mathrm{e}-05$ & 0.0103 & 0.0189 & 0.0275 & $0.1 \mathrm{e}-5$
\end{tabular}

In addition to detailed statistical tests and classification steps, statistical box-plots of brain network measures, estimated using PC, were also shown in Figure 2. The graphical representation of the estimations showed that increased segregation (in terms of $T$ and $C C$ ) and increased resilience (in terms of $r$ ) were observed in post-treatment recordings. Besides, treatment with OROSMPH caused decrease in integration (in terms of $G E$ ) and modularity $(Q)$.

To present visual representation of brain network analysis, grand average adjacency matrixes were shown in Figure 3. For each participant, dependency matrix was computed for 30 number of non-overlapped short segments of 2 sec. Threshold was individually applied to each dependency matrix in transforming the binomial values into binary levels. The number of patients were 18. Thus, total number of resting-state eyes-opened adjacency matrixes was 540 (30x18). In both pre- and post-treatment periods, grand average of adjacency matrixes was obtained over 540 estimations. This figure showed that functional brain network was changed clearly, however, regional neural activations can not be 


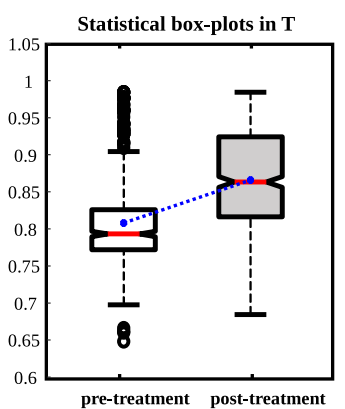

(a)

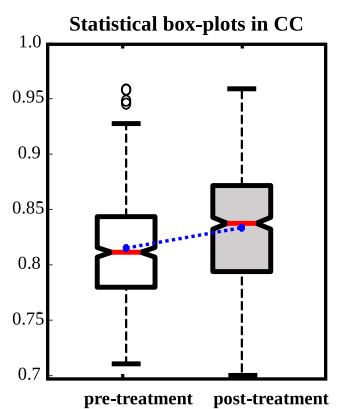

(b)

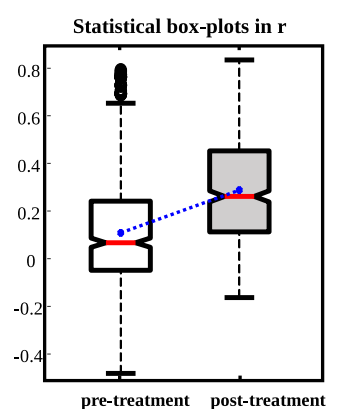

(c)

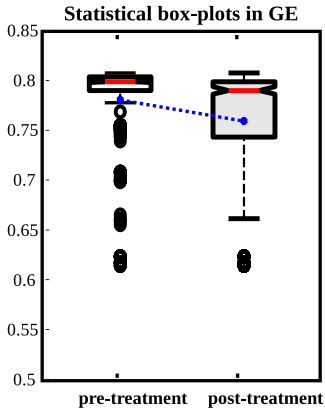

(d)

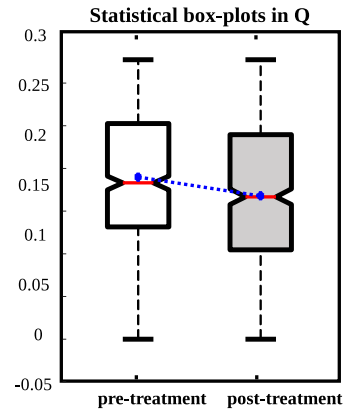

(e)

Fig. 2 Statistical box-plots of brain network measures as $T$ (a), $C C$ (b), $r$ (c), $G E$ (d) and $Q$ (e) extracted from adjacency matrices of 30 separate EEG segments for each individual in each group

related to grand average adjacency matrix. Averaging was not used in either statistical test or two-class classifications.

Combining step-wise logistic regression modelling results and statistical test results with graphical statistical box-plot, two-class classification was also performed into several feature set design in order to be sure about alterations in brain mechanism induced by OROS-MPH. Thus, the feature sets were organized inform,

- 1st design included the features selected from estimations in a single brain network index,

- 2nd design included two applications for (1) the features selected from estimations in only increased brain network indices ( $T, C C$ and $r$ ) and, (2) the features selected from estimations in decreased brain network indices $(G E$ and $Q)$,

- 3rd design included the features selected from the estimations in brain network indices included in step-wise logistic regression modelling ( $T, C C$, $r$ and $G E)$ 


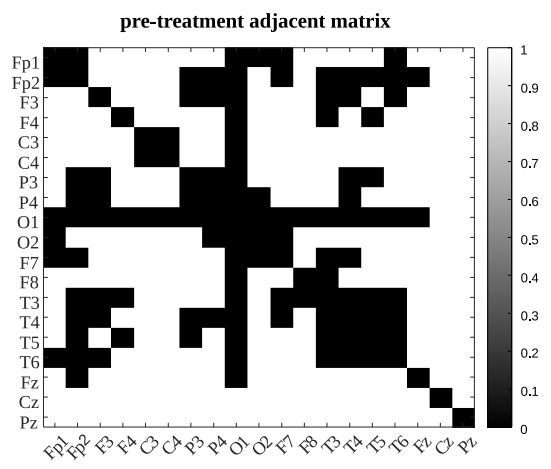

(a)

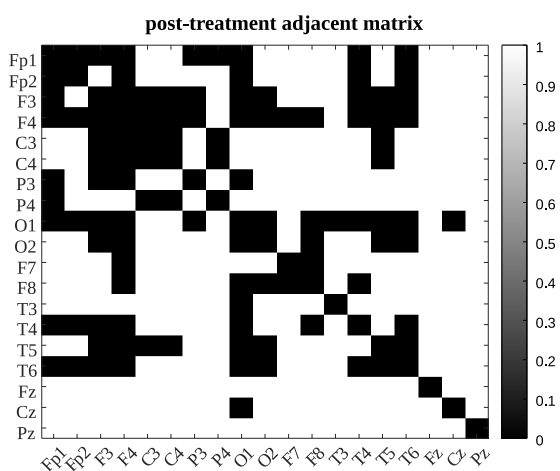

(b)

Fig. 3 The grand average adjacency matrixes extracted from hemispheric dependency estimations by PC in (a) pre- and (b) post-treatment periods in patients

The corresponding classification performances were compared to each other in Table 6 in terms of CA (\%). When the groups were classified with respect to single brain network index, the lowest $\mathrm{CA}$ was generated by modularity estimations $(Q)$. When the brain network indices, increased in post-treatment recordings, were combined as the features, relatively high $\mathrm{CA}$ was obtained in comparison to the combination of the other indices, decreased in posttreatment recordings. Comparing tables 3 and 6 , excluding modularity index $(Q)$ in the features provided the highest classification performance in discriminating groups. In other words, combination of both segregation ( $T$ and $C C$ ) and resilience $(r)$ as the features provided the best results instead of all indices $(T, C C, r, G E, Q)$.

Table 6 SVM classification performances in discriminating the groups with respect to different feature set designs

\begin{tabular}{|c|c|c|c|c|c|}
\hline & Segregation & Segregation & Resilience & Integration & Modularity \\
\hline & $T$ & $C C$ & $r$ & $G E$ & $Q$ \\
\hline alteration & increase & increase & increase & decrease & decrease \\
\hline $\mathrm{RBF}$ & 68.79 & 71.88 & 63.61 & 70.55 & 60.64 \\
\hline Gaussians & 67.96 & 66.20 & 63.79 & 69.62 & 59.53 \\
\hline RBF & \multirow{2}{*}{\multicolumn{3}{|c|}{$\begin{array}{r}83.79(T, C C \text { and } r) \\
81.29(T, C C \text { and } r)\end{array}$}} & \multirow{2}{*}{\multicolumn{2}{|c|}{$\begin{array}{l}77.22(G E \text { and } Q) \\
72.59(G E \text { and } Q)\end{array}$}} \\
\hline Gaussians & & & & & \\
\hline $\begin{array}{l}\text { RBF } \\
\text { Gaussians }\end{array}$ & \multicolumn{5}{|c|}{$81.01(T, C C, r, G E)$} \\
\hline
\end{tabular}




\section{Discussion and Conclusion}

In the present study, resting-state eyes-opened EEG series were recorded from 18 boys diagnosed with ADHD-C before medication phase. They had a specified treatment as OROS-MPH for a month. Then post-treatment resting-state eyes-opened EEG series were also recorded from them. Graph Theoretic brain network indices were estimated from both pre- and post-treatment related EEG recordings with respect to un-directed weighted adjacency matrixes that are extracted from brain dependency and connectivity estimations by PC, SC, PLV, PLI, DTF and PDC. In obtaining adjacency matrixes, threshold was $60 \%$ of maximum value in dependency matrix of interest in each short EEG segment of $2 \mathrm{sec}$ over trial of $1 \mathrm{~min}$.

The resulting estimations were investigated through both classifications with SVMs and statistical tests including one-way Anova and pair-wise multiple comparison. Finally, graphical box-plots of brain network indices were shown in pre- and post-treatment recordings in accordance with $\mathrm{PC}$ that provided superior results. Regarding step-wise logistic regression modelling, brain modularity (Q) is found to be less sensitive to treatment with OROS-MPH. The highest classification performance of $83.79 \%$ was obtained when three brain network indices ( $T, C C$ and $r$ ) were combined as feature set. The lowest statistical p-values were obtained for these three indices.

Combining quantitative results reveal that functional brain segregation $(T$ and $C C$ ) and resilience $(r)$ indices are mostly influenced by OROS-MPH, while integration and modularity indices are less affected by medication in boys with ADHD-C. However, the statistical box-plots on brain network indices showed the following brief results:

increased $T$ indicates stronger connectivity of cortical regions to neighbors, increased $C C$ indicates increased efficiency of information flow,

increased $r$ indicates increased number of interconnected neural populations

thereby forming a resilient core to spread the info over the whole cortex, decreased $G E$ indicates decreased number of disconnected nodes, decreased $Q$ indicates decreased probability in determining cortical communication net into independent sub-nets.

From methodological point of view, statistical correlation method, PC provided the best results to compute brain dependency values that provide strong estimations in brain network measures from resting state eyes-opened surface EEG series. PC is found to be superior to both phase domain functional synchronization methods (PLV, PLI) and frequency domain effective connectivity methods (DTF, PDC).

From computational psychiatric point of view, quantitative visual results reveal that OROS-MPH clearly influence functional brain segregation and resilience in boys with ADHD-C. In detail, OROS-MPH provide the followings: (1) functional connectivity between cortical regions becomes more stronger, (2) neuronal undirected information flow becomes more efficient and (3) the number of interconnected regional neural populations that are required to 
form a resilient that provides information spread across the whole cortex. In future work, we are plan to record emotional EEG series from three groups of the patients (ADHD-I, ADHD-HI, ADHD-C) in response to affective static pictures (unpleasant vs pleasant) in both pre- and post- treatment periods in estimating brain network measures.

\section{Declaration of Competing Interest}

The authors declare that they have no conflict of interest.

\section{Ethical Approval}

Ethical approval for this study was obtained from the Ethics Committee of Gazi University, Medical Faculty (Approval Number: 2011/25, Date:25.05.2011). Written consents of the patients and their parents were obtained.

\section{Role of funding}

Authors of this article declare that they have no funding support.

\section{Authors Contributions}

Author FHÇ, MU, ASG, YI and ZB designed the study and wrote the protocol. Author SA provided the novelty of this study, analyzed the data and wrote the paper.

\section{Consent to Publish}

All authors contributed to and have approved the manuscript for publication.

\section{Data Availability Statement}

EEG data is available upon request. The second author is responsible for analysis on the data.

\section{References}

1. American Psychological Association. (2001). Publication manual of the American, Psychological Association (5th ed.). Washington: APA

2. Castellanos, F.X., Margulies, D.S., et al. (2008) Cingulate-precuneus interactions: a new locus of dysfunction in adult attention-deficit/hyperactivity disorder, Biol Psychiatry, 63(3):332-337 
3. Fair, D.A., Posner, J., Nagel, B.J., Bathula, D. et al. (2010) Atypical default network connectivity in youth with attention-deficit/hyperactivity disorder, Biol Psychiatry, 68(12):1084-1091

4. Qiu, M.G., Ye, Z., Li, Q.Y., et al. (2011) Changes of brain structure and function in ADHD children, Brain Topogr, 24(3-4):243-252

5. Alba, G., Pereda, E., Mañas, S., Mendez, L.D. et al. (2016) The variability of EEG functional connectivity of young ADHD subjects in different resting states, Clin Neurophysiology, 127(2):1321-1330, doi:10.1016/j.clinph.2015.09.134.

6. Gonzalez, J.J., Mendez, L.D., Manas, S. et al. (2013) Performance analysis of univariate and multivariate EEG measurements in the diagnosis of ADHD, Clin Neurophysiology, 124:1139-1150.

7. Barry, R.J., Clarke, A.R., Hajos, M., et al. (2011) EEG coherence and symptom profiles of children with attention-deficit/hyperactivity disorder, Clin Neurophysiol, 122(7):13271332

8. Dupuy, F.E., Clarke, A.R., Barry, R.J. et al. (2010) EEG coherence in children with attention-deficit/hyperactivity disorder: differences between good and poor responders to methylphenidate, Psychiatry Res, 180(2-3):114-119

9. Murias, M., Swanson, J.M., Srinivasan, R. (2007) Functional connectivity of frontal cortex in healthy and ADHD children reflected in EEG coherence. Cereb Cortex, 17(8):1788-99, doi:10.1093/cercor/bhl089.

10. Ochsner, K.N., Gross, J.J. (2008) Cognitive emotion regulation: insights from social cognitive and affective neuroscience, Current Directions in Psychological Science, 17 $153-158$.

11. Gross, J.J. (2002) Emotion regulation: affective, cognitive, and social consequences, Psychophysiology, 39(3):281-91.

12. X. Liao, A.V. Vasilakos, Y. He, (2017) Small-world human brain networks: Perspectives and challenges, Neuroscience \& Biobehavioral Reviews, 77:286-300, doi:10.1016/j.neubiorev.2017.03.018.

13. Sporns O. (2018). Graph theory methods: applications in brain networks, Dialogues in Clinical Neuroscience, 20(2):111-121, doi:10.31887/DCNS.2018.20.2/osporns

14. Ren, Y., Cong, F., Ristaniemi, T. et al. (2019) Transient seizure onset network for localization of epileptogenic zone: effective connectivity and graph theory-based analyses of ECoG data in temporal lobe epilepsy. $J$ Neurology, 266:844-859, doi:10.1007/s00415019-09204-4.

15. Franciotti R, Falasca NW, Arnaldi D, Famà F, et al. (2019) Cortical Network Topology in Prodromal and Mild Dementia Due to Alzheimer's Disease: Graph Theory Applied to Resting State EEG, Brain Topography, 32(1):127-141, doi:10.1007/s10548-018-0674-3.

16. Kim,J., Lee, W.G., Park, S., Park, K.M. (2020) Can we predict drug response by functional connectivity in patients with juvenile myoclonic epilepsy?, Clin Neurology and Neurosurgery, doi:10.1016/j.clineuro.2020.106119.

17. Ostojic, S., Brunel, N., Hakim, V. (2009). How connectivity, background activity, and synaptic properties shape the cross-correlation between spike trains. J. Neuroscience, 29:10234-10253.

18. Scholvinck, M.L., Friston, K.J., Rees, G. (2011). The influence of spontaneous activity on stimulus processing in primary visual cortex. NeuroImage, 80, 297-306.

19. Zalesky, A., Fornito, A., Bullmore, E., (2012). On the use of correlation as a measure of network connectivity. NeuroImage, 60(4):2096-2106.

20. Sarmukadam,K., Bitsika, V., Sharpley, C.F. et al. (2020) Comparing different EEG connectivity methods in young males with ASD, Behavioural Brain Research, 383:112482, doi:10.1016/j.bbr.2020.112482.

21. Fries P. (2005) A mechanism for cognitive dynamics: neuronal communication through neuronal coherence. Trends in cognitive sciences, 9:474-480, doi:10.1016/j.tics.2005.08.011

22. Lachaux, J. P., Rodriguez, E., Martinerie, J., Varela, F. J. (1999). Measuring phase synchrony in brain signals. Human brain mapping, 8(4):194-208, doi:10.1002/(sici)10970193

23. Stam, C. J., Nolte, G., Daffertshofer, A. (2007). Phase lag index: assessment of functional connectivity from multi-channel EEG and MEG with diminished bias from common sources. Human Brain Mapping, 28(11):1178-1193, doi:10.1002/hbm.20346 
24. Lin,L.C., Ouyang, C.S., Chiang, C.T. et al. (2018) Cumulative effect of transcranial direct current stimulation in patients with partial refractory epilepsy and its association with phase lag index-A preliminary study, Epilepsy 86 Behavior, 84:142-147, doi:10.1016/j.yebeh.2018.04.017.

25. Orekhova E.V., Elsabbagh M., Jones E., Dawson G., et al. (2014) EEG hyperconnectivity in high-risk infants is associated with later autism. J. Neurodev. Disord., 6(1):40, doi:10.1186/1866-1955-6-40.

26. van de Pol L.A., van't Westende C., Zonnenberg I., Koedam E., et al. (2018). Strong relation between an EEG functional connectivity measure and postmenstrual age: A New potential tool for measuring neonatal brain maturation, Frontiers in Human Neuroscience, 12, 286. doi:10.3389/fnhum.2018.00286

27. Olejarczyk, E., Jernajczyk, W. (2017). Graph-based analysis of brain connectivity in schizophrenia, PloS one, 12(11):e0188629. doi:10.1371/journal.pone.0188629

28. Lung-Chang L., Chen-Sen O., Ching-Tai C., Rei-Cheng Y., et al. (2018) Cumulative effect of transcranial direct current stimulation in patients with partial refractory epilepsy and its association with phase lag index-A preliminary study, Epilepsy \& Behavior 84:142-147, doi:10.1016/j.yebeh.2018.04.017.

29. Cocchi L, Bramati IE, Zalesky A, Furukawa E, et al. (2012) Altered functional brain connectivity in a non-clinical sample of young adults with attention-deficit/hyperactivity disorder. J Neurosci., 32(49):17753-61. doi:10.1523/JNEUROSCI.3272-12.2012.

30. Mills, K.L., Bathula, D., Dias, T.G., Iyer, S.P., et al. (2012) Altered cortico-striatalthalamic connectivity in relation to spatial working memory capacity in children with ADHD. Front Psychiatry, doi:10.3389/fpsyt.2012.00002.

31. Turgay, A. (1994) Disruptive behavior disorders child and adolescent screening and rating scales for children, adolescents, parents and teachers. West Bloomfield (Michigan): Integrative Therapy Institute Publication.

32. Ercan E. (2001) Development of a test battery for the assessment of attention deficit hyperactivity disorder. Turk J Child Adolesc Psychiatry, 8:132-44.

33. Kaufman, J., Birmaher, B., Brent, D., et al. (1997) Schedule for affective disorders and schizophrenia for school-age children-present and lifetime version (K-SADS-PL): initial reliability and validity data. J. of the American Academy of Child $\&$ Adolescent Psychiatry, 36(7):980-8.

34. Gokler B. (2004) Reliability and validity of schedule for affective disorders and Schizophrenia for school age children-present and lifetime version-Turkish version (KSADS-PL-T)[in Turkish]. Turk J Child Adolesc Mental Health. 11:109-16

35. Liu, T., Chen, Y., Lin, P., Wang, J. (2015) Small-world brain functional networks in children With Attention-Deficit/Hyperactivity Disorder revealed by EEG synchrony, Clin EEG Neurosci., 46(3):183-91.

36. Huang, D., Ren, A. et al. (2016) Combining partial directed coherence and Graph Theory to analyse effective brain networks of different mental tasks, Front. Hum. Neurosci., doi:10.3389/fnhum.2016.00235

37. Olejarczyk, E., Zuchowicz, U., Wozniak-Kwasniewska, A. et al. (2020) The impact of repetitive transcranial magnetic stimulation on functional connectivity in major depressive disorder and bipolar disorder evaluated by directed transfer function and indices based on Graph Theory, Int J of Neral Systems, doi:10.1142/S012906572050015X

38. Vázquez, M.A., Maghsoudi, A., Marifio, I.P. (2021) An interpretable machine learning method for the detection of schizophrenia using EEG signals, Front. Syst. Neurosci. doi:10.3389/fnsys.2021.652662

39. Cao, Q., Shu, N., An, L., Wang, P., et al. (2013) Probabilistic diffusion tractography and graph theory analysis reveal abnormal white matter structural connectivity networks in drug-naive boys with attention deficit/hyperactivity disorder, J Neuroscience, 33(26):10676-8\%.

40. Fraga Gonzalez, G., Smit, D., van der Molen, M., Tijms, J., et al. (2018). EEG Resting State Functional Connectivity in Adult Dyslexics Using Phase Lag Index and Graph Analysis. Frontiers in Human Neuroscience, 12:341. doi:10.3389/fnhum.2018.00341

41. Liu W., Zhang C., Wang X., Xu J., Chang Y., Ristaniemi T., Cong F. (2020). Functional connectivity of major depression disorder using ongoing EEG during music perception. Clin Neurophysiology, 131(10):2413-2422. doi:10.1016/j.clinph.2020.06.031. 
42. Rubinov M., Sporns O. (2010) Complex network measures of brain connectivity: uses and interpretations. Neuroimage, 52(3):1059-69. doi:10.1016/j.neuroimage.2009.10.003.

43. Goni J., Avena-Koenigsberger A., Velez de Mendizabal N., et al. (2013) Exploring the morphospace of communication efficiency in complex networks. PLoS One, 8(3):e58070. doi:10.1371/journal.pone.0058070.

44. Latora, V., Marchiori, M. (2001) Efficient behavior of small-world networks, Phys. Rev. Letters, 87(19):198701

45. Wu, C. F. J., and M. Hamada. Experiments: Planning, Analysis, and Parameter Design Optimization, 2000.

46. Kaminski $M$, Ding $M$, Truccolo $W$, Bressler S. (2001) Evaluating causal relations in neural systems: Granger causality, directed transfer function and statistical assessment of significance, Biol Cybern, 85:145-5\%.

47. He, B., Dai, Y., Astolfi, L., Babiloni, F., et al. (2011) eConnectome: A MATLAB Toolbox for Mapping and Imaging of Brain Functional Connectivity. J. of Neuroscience Methods, 195:261-269. 